\title{
Long Term Outcome of Juvenile Onset Recurrent Respiratory Papillomatosis in University Kebangsaan Medical Centre
}

\author{
Goh Bee See ${ }^{1}$, Nadhirah Mohd Shakri', Jenny Loh Chen Nee ${ }^{2}$ \\ 1 Department of Otorhinolaryngology, University Kebangsaan Malaysia Medical Centre, Kuala Lumpur, Malaysia \\ 2 Department of Otorhinolaryngology, Pantai Hospital Ayer Keroh, Melaka, Malaysia \\ Goh Bee See, ORCID: 0000-0002-5535-2930 \\ Nadhirah Mohd Shakri, ORCID: 0000-0003-4939-2562 \\ Jenny Loh Chen Nee, ORCID: 0000-0002-7930-7528
}

\begin{abstract}
Objective: To review the long term outcome of juvenile onset recurrent respiratory papillomatosis (JORRP) in University Kebangsaan Medical Centre (UKMMC).

Methods: A sixteen-year retrospective study - from January 2002 to June 2019 - of patients who presented with JORRP at our hospital was carried out.

Results: Nineteen cases were identified with the youngest age of presentation being eight months old. The mean follow-up time was 4.8 years. All patients had glottic papilloma, ten had multi-site involvement, namely at the subglottic, trachea, oropharynx and one case of distal spread to the lungs. The majority $(71.4 \%)$ of patients with tracheostomy had extralaryngeal spread and shorter surgical intervals (a mean of 3.2 procedures per year). All our patients were treated surgically. Two patients
\end{abstract}

with aggressive disease received a trial of the adjuvant therapies Gardasil and Cidofovir. However, no improvement was observed.

Conclusion: The worst prognosis is seen in youngeronset JORRP. Surgery is the mainstay of treatment and patients with aggressive disease may need adjuvant therapy. Unfortunately, there is a lack of a standard protocol for adjuvant therapies. Tracheostomy increases the risk of extralaryngeal spread and aggressive disease, and thus should be reserved for patients with severe airway compromise.

Keywords: Human papillomavirus infection, respiratory tract, pediatrics, tracheostomy. 


\section{Introduction}

Recurrent respiratory papillomatosis (RRP) is a rare disease caused by human papilloma virus (HPV) types 6 and $11 .^{[1-3]}$ Patients presenting with this disease at 12 years of age or younger are diagnosed with juvenile-onset recurrent respiratory papillomatosis (JORRP). In the United States, the incidence rate of JORRP is reported as 4.3 per 100,000 children. ${ }^{[4]} \mathrm{HPV}$ has been hypothesized to be transmitted vertically in utero during labour. The reservoir of infection lies within the genital tract with a predilection towards the squamocolumnar junction. ${ }^{[1]}$ However, a recent study also suggests hematogenous in utero infection. ${ }^{[5]} \mathrm{RRP}$ is characterized by recurrent exophytic papillomas of the epithelial mucosa in the respiratory tract, most commonly in the larynx. Repetitive debulking for airway control is the current standard management of RRP. Adjuvant therapy may be administrated in aggressive disease, although there is a lack of a standard protocol. ${ }^{[2]}$ In cases with potential airway occlusion, a tracheostomy may be needed. However, tracheostomy increases the risk of rapid colonization and serves as a conduit for distal spread to the tracheobronchial tree. ${ }^{[1]}$ Thus, it is reserved for patients with airway compromise where multiple surgeries have failed. This paper aimed to review the long-term outcome of JORRP at the University Kebangsaan Medical Centre (UKMMC).

\section{Materials and Methods}

We conducted a retrospective review of nineteen patients treated for JORRP at the UKMMC between January 2002 and June 2019. Patient files were traced from the medical records office and the hospital's computer system. Patient ethnicity, age, gender, number of surgical procedures, course of the disease, sites of involvement and tracheostomy prevalence were analyzed. Their progress and outcomes were also documented in this series.

\section{Results}

There were twelve male and seven female patients, with a male-to-female ratio of 1.71. Eleven of the patients were Chinese, six were Malay and two were foreigners (Table 1). The age of presentation ranged from two to eight years old (mean of 3.8), except for one patient who was treated for JORRP as early as eight months old. All our patients were delivered vaginally and eight $(42.1 \%)$ were firstborn children. One of the mothers had maternal warts and none of our patients were born to a teenage mother. The mean follow-up time in our series was 4.8 years (range of 1 to 14 years) and the average number of past procedures was 5.5.

\section{Sites of involvement}

All patients had glottis involvement. Thirteen patients $(68.4 \%)$ had multiple sites of involvement, namely tonsils, epiglottis, post cricoid, pyriform fossa, oesophageal opening, subglottis and trachea (Figure 1). One case of distal spread to the lungs was seen in patient 14 . Another patient (patient 13) had nasal septum involvement.

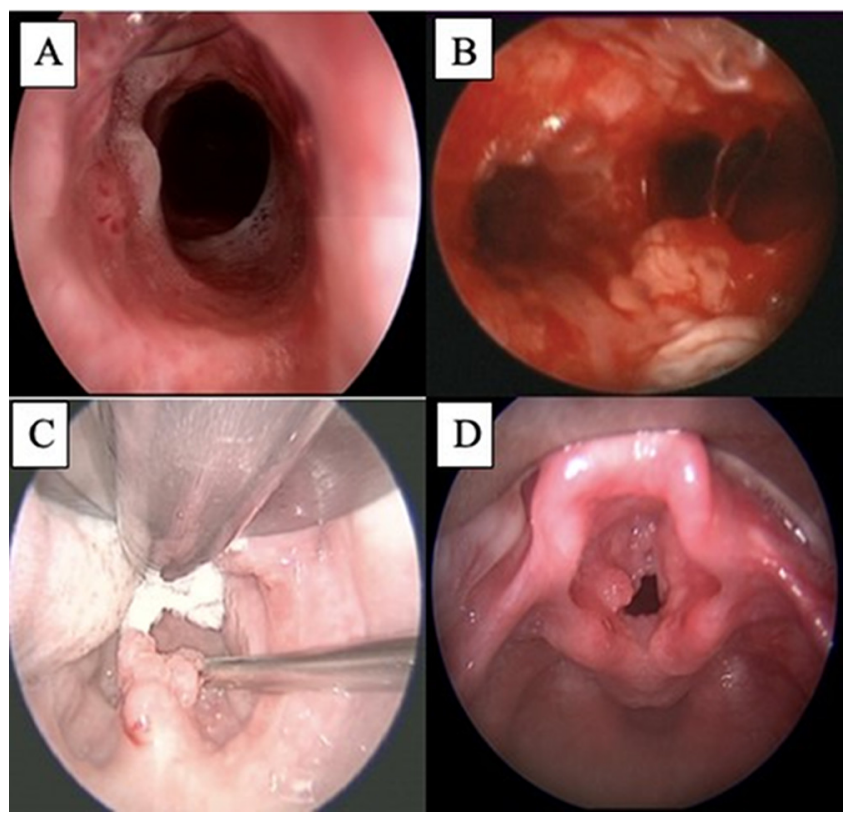

Figure 1. Multiple sites of extra-laryngeal involvement in lower airways (A and B), oropharynx (C) and supraglottis (D).

\section{Tracheostomy and extra laryngeal involvement}

Seven $(36.8 \%)$ of our cases required a tracheostomy to relieve upper airway obstruction due to extensive disease. The presence of tracheostomy was related to more aggressive disease, shorter surgical intervals and extralaryngeal spread (Table 1). Patients who had tracheostomy required more procedures per year (mean of 3.2) compared to those without tracheostomy (mean of 1.5).

Extralaryngeal involvement was observed in the majority $(71.4 \%)$ of our patients with tracheostomy mainly at the peristoma, tracheal wall and subglottis (Figure 2). Patient 13 had been treated for JORRP since 2004 at the age of 2 years old. A tracheostomy was performed two years later, 


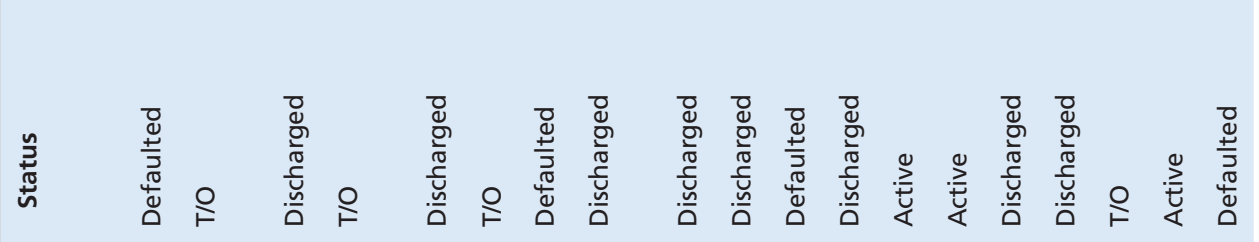

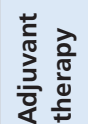

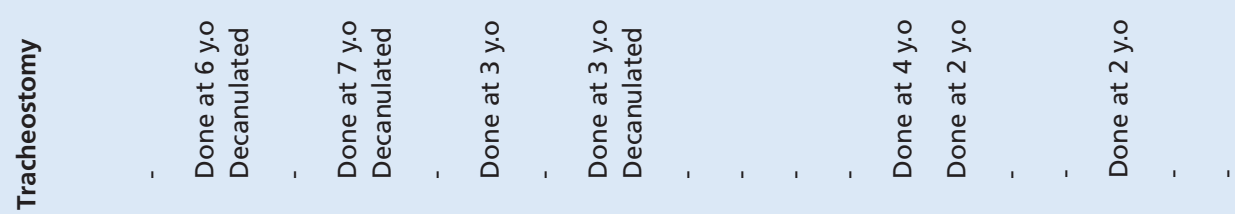

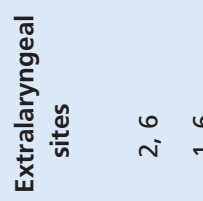

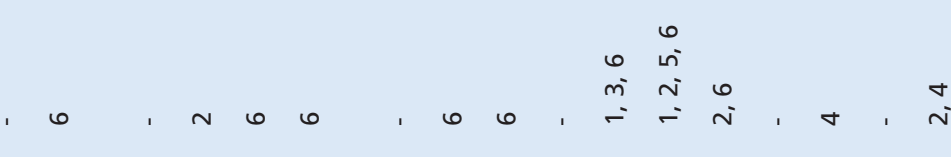

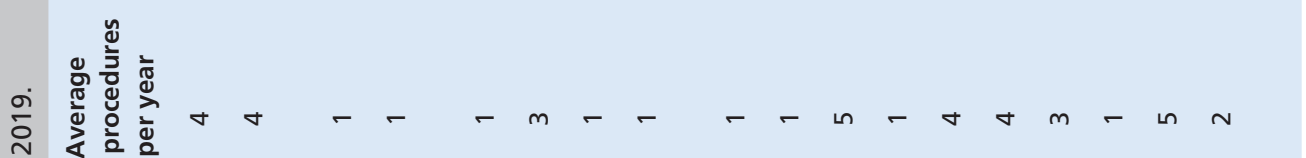

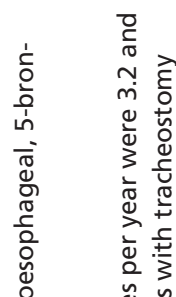

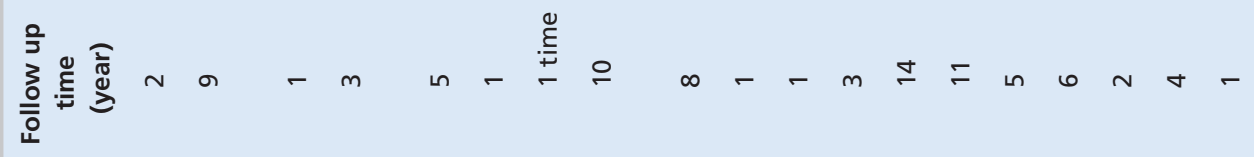

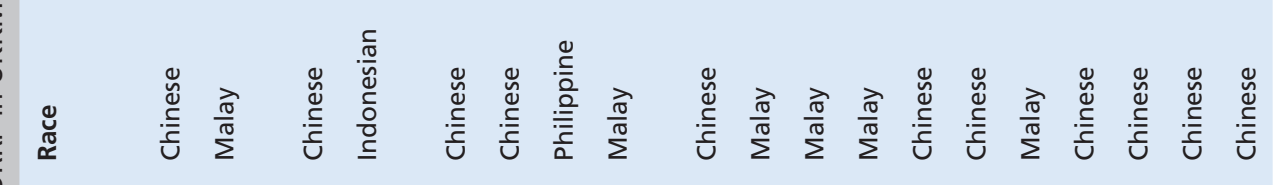




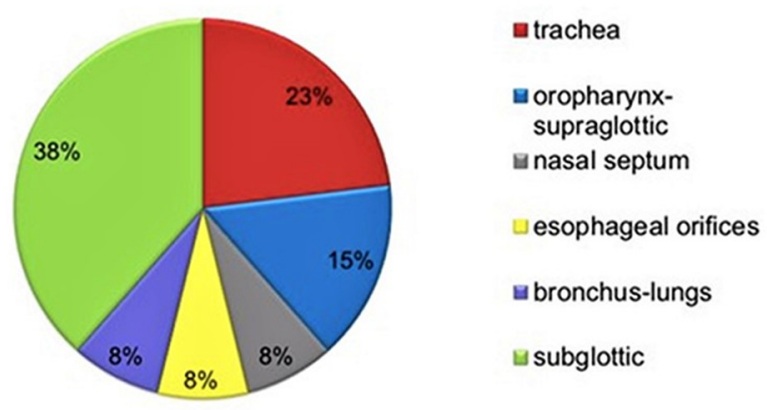

Figure 2. Distribution sites of extra-laryngeal involvement in patients with tracheostomy. The subglottic and trachea are the most common sites for extra-laryngeal spread.

and he was referred to our centre in 2007 at the age of five. He had extralaryngeal involvement at the trachea and nasal septum (Table 1). Patient 14, with the youngest age of presentation as mentioned, underwent a tracheostomy for recurrent severe upper airway obstruction as early as two years old (Figure 3). He had extralaryngeal papillomatosis over the trachea and lungs. Thorax computed tomography revealed bronchial papillomatosis of the right lung in the middle lobe. The latest chest X-ray in 2019 showed increasing cavitary lesions in the middle and lower zones of

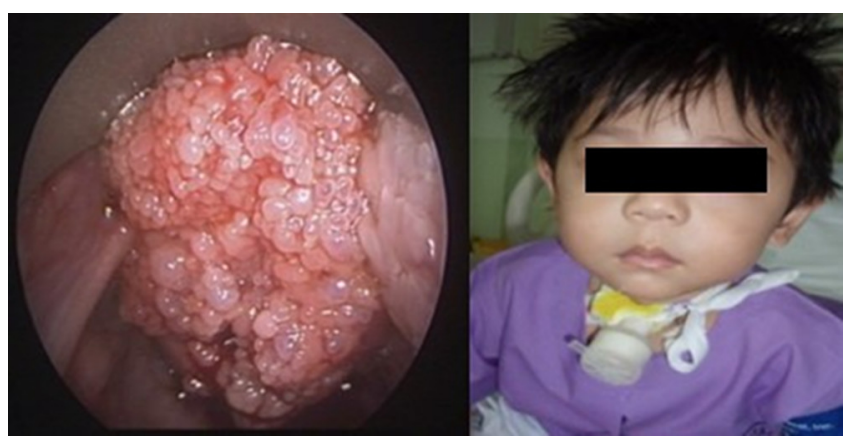

Figure 3. Patient with extensive papillomatous lesion occupying the airway requiring tracheostomy.

both lungs. With the earliest age of presentation, multiple sites of involvement and more than 10 years follow-up with a history of 67 procedures, he was obviously the patient with the most fulminant disease.

Extralaryngeal involvement was also observed in three patients without tracheostomy. Patient 15 , who was diag- nosed with JORRP at two-years-old, had subglottis, supraglottis and posterior pharyngeal wall involvement. Patient 19 had tonsils, palate and oesophageal orifice involvement. Unfortunately, he defaulted on the follow-up visit after two debulking surgeries.

\section{Adjuvant therapy}

Patient 13 was given a trial of adjuvant therapy with three doses of Gardasil injections in July 2017, September 2017 and March 2018. However, this patient still had recurrence after completing adjuvant therapy.

Patient 14 received a trial of intralesional Cidofovir injections eight times at the age of three from October 2009 until August 2010. He also received three doses of Gardasil injections in 2017. Unfortunately, no improvement was observed in his recurrence rate or surgical interval after completing both adjuvant therapies.

\section{Progress and outcome}

Patient 14 had dysplasia of laryngeal papillomas two years after the diagnosis of JORRP was made. Regular six-month biopsies of the laryngeal papilloma persistently reported as focal mild dysplasia for five years from October 2009 until September 2014. However, subsequent biopsies up until June 2019 were consistent with laryngeal papilloma without dysplasia.

Eight patients (42.1\%) had spontaneous remission after multiple procedures to debulk the papilloma and were discharged. Three patients (15.7\%) were stabilized and transferred back to the referring hospital (Figure 4). Patient 15 had multiple sites of involvement and had undergone 29 procedures in five years between 2011 and 2014 before remission was noted at the age of six. She was discharged with good voice quality. Five patients $(26.3 \%)$ did not attend the follow-up appointments, including patient 19 who had multiple sites of involvement.

Four patients with tracheostomy managed to be decannulated and discharged or transferred back to the referring hospital. However, patient 2 needed a second tracheostomy due to extensive disease. Subsequently, her condition was stabilized and she was transferred back to the referring hospital. Patient 13 managed to be decannulated, however, he is still undergoing periodic debulking surgeries at UKMMC, together with patients 14 and 18. Patient 14, who has the most fulminant disease, still requires a tracheostomy due to extensive laryngeal, tracheal and lung involvement. There was no mortality in this series. 


\section{Discussion}

Recurrent respiratory papillomatosis caused by HPV types 6 and 11 is the most common benign neoplasm of the larynx. ${ }^{[1-3]}$ The course of JORRP is unpredictable as some patients present with aggressive disease, requiring multiple surgical debulking and hospitalizations. It has been hypothesised that HPV is transmitted vertically from mother to neonate during passage through the birth canal. However, this route has not been shown conclusively as the only mechanism of HPV infection. A systematic review in 2010 by Winckworth et al ${ }^{[6]}$ showed there was no statistically significant difference between HPV transmission rates through vaginal delivery and caesarean section. Another recent study reported that HPV DNA was detected in the cord blood of neonates of asymptomatic HPV-positive mothers, which supports haematogenous in utero spread. [5] The clinical triad of a firstborn child delivered vaginally to a teenage mother has been linked to JORRP. Despite all our patients being born vaginally, none fulfilled all the components of the triad.

In 2014, Omland et al [7] reported that age of onset is associated with more aggressive disease in JORRP. In this study, aggressive disease was defined as a distal spread, a tracheostomy, four surgical operations annually or more than 10 surgeries in total. Our youngest patient with the most fulminant disease (patient 14) presented with aphonia when he was eight months old. Male preponderance is observed in our series, notwithstanding the review by Long et al ${ }^{[8]}$ at UKMMC from 1996 to 2001, which reported the majority of JORRP incidence among females (a ratio of 5:1).

Papillomas can be found anywhere in the aerodigestive tract with the most common locations found at the junction of the squamous and ciliary epithelium and larynx..$^{[2,3]}$ In the present study, all the patients had glottis involvement. Other predominant sites were the limen vestibuli, the nasopharyngeal surface of the soft palate, the midzone of the laryngeal surface of the epiglottis, the upper and lower margins of the ventricle, the undersurface of the vocal folds, the carina and the bronchial spurs.

Surgical debulking is the standard care in the management of RRP. The objectives of surgery are to preserve adequate voice quality and airway patency. ${ }^{[4]}$ Surgery is performed under general anesthesia with an endotracheal tube (ETT). We also use tubeless jet ventilation, or apnoeic ventilation, particularly if the papilloma is at the posterior commissure, which is obscured by the ETT. Once the diagnosis is confirmed, we usually remove the papillomatous lesions via CO2 ablation or the debrider technique depending on the location and extent of the disease and the facilities available at that time. Microdebriders can be used in combination with lasers, with microdebriders first removing the bulk of the papilloma, and lasers then providing hemostatic ability and more precise treatment of sessile disease. If the papilloma involves subglottic and upper tracheal tissue, we use a subglottic scope for direct visualization, and then ablate the lesion with a laser. Cold instruments, such as cupped forceps, are mainly for biopsy purposes. Complete eradication is not necessary, as HPV can be dormant even in the 'normal-looking' mucosa adjacent to papillomas. Extensive excision of papilloma from sites that are not contributing to airway or voice-related goals, has not been shown to reduce recurrence rates. ${ }^{[4]}$ Extensive excision should be avoided at the anterior commissure to prevent webbing and scarring and limit dysphonia.

A tracheostomy creates an extra squamocolumnar junction for papillomas to breed and may be the cause of extralaryngeal spread. ${ }^{[1]}$ It is usually reserved for patients with aggressive disease, which has the potential to occlude the airway. Due to the affiliation of HPV with the squamocolumnar junction, this factor has been related to the aggressive nature of patients with tracheostomy as compared to patients without tracheostomy. Most of our patients underwent debulking surgeries without the need for a tracheostomy. Of the seven patients for whom a tracheostomy was performed, the majority (71.4\%) developed extralaryngeal spread at the peristoma, subglottis, tracheal wall and, in one case, the lungs. All our patients with tracheostomy managed to be decannulated or stabilised and were transferred back to the referring hospital, except for patient 14. A possible reason for the failure of decannulation was the extensive tracheal and lung involvement, which relates to aggressive disease.

The genetic differences between HPV 11 and HPV 6, which could explain the higher risk of malignant transformation in HPV 11 patients, are still unknown. Thus, regular histopathological examinations must be performed in JORRP patients. At our centre, we perform regular biopsies of papillomatous lesions every six months. Patient 14 had focal low-grade dysplasia of papilloma in the larynx for five years from August 2009 until September 2014. However, subsequent biopsies performed until now showed no dysplasia or malignant transformation. The focal dysplastic papillomas were probably completely removed during 
surgery. As far as we know, there have been no studies that have reported such histopathological changes from dysplasia back to 'normal' papilloma.

Although surgery is the primary treatment for RRP, approximately $20 \%$ of RRP patients require adjuvant therapy to control the disease. ${ }^{[10]}$ However, limited data exist concerning the optimal dosage, efficacy and possible adverse effects. Indications for adjuvant therapy are the need for more than four surgical procedures per year, rapid regrowth of papillomas with airway compromise or distal multi-site spread of disease. ${ }^{[2]}$ Interferon (IFN) is one of the first systemic adjuvant treatments used to manage RRP. Its exact action against RRP is unknown, and the clinical efficacy of IFN in the treatment of RRP is controversial. One of our patients that had received IFN therapy had a longer interval between surgeries, however, the recurrence was still the same. IFN therapy is rarely used due to the emergence of intralesional adjuvants, such as Cidofovir and Bevacizumab.

Cidofovir acts by inhibiting DNA polymerization and is known to display antiviral activity against a variety of Herpes viruses. ${ }^{[11]}$ In 2003, Pransky et al ${ }^{[12]}$ reported a remarkable decrease in the total number of surgical procedures per year and an increase in the interval between procedures in children with RRP who were treated with intralesional Cidofovir injections. Unfortunately, case reports, although limited, have reported malignant transformation associated with Cidofovir use for RRP in humans, but there is a lack of evidence of controlled trials to make reliable conclusions. ${ }^{[1]}$ The US Food and Drug Administration has only approved Cidofovir for the treatment of cytomegalovirus retinitis in patients with acquired immunodeficiency disease. ${ }^{[1]}$ Bevacizumab is a recombinant monoclonal humanised antibody that blocks angiogenesis by inhibiting human vascular endothelial growth factor A (VEGF-A). ${ }^{[13]}$ In 2013, Rogers ${ }^{[13]}$ et al performed a pilot study on ten JORRP patients by giving three intralesion-al Bevacizumab injections at a concentration of $2.5 \mathrm{mg} /$ $\mathrm{mL}$ every two to three weeks. No adverse effects were observed, and the results showed that children who were treated with intralesional Bevacizumab injections required four fewer surgical procedures per year and had longer intervals between surgeries, which improved their quality of life. Patient 14, who had pulmonary involvement, received a trial of intralesional Cidofovir injections, but no improvement was observed. $\mathrm{He}$ was offered Bevacizumab, but it was refused by his parents.
The latest and most exciting development in the management of RRP is prevention through HPV vaccination. The quadrivalent human papillomavirus (qHPV) vaccine, Gardasil, works against both low-risk HPV types 6 and 11 and high-risk HPV types 16 and 18 . The excellent effects of Gardasil were demonstrated in the study of Kin Cho Goon et al ${ }^{[14]}$ in 2017 who reported a more than seven-fold decrease in incidence rates of papillomatosis requiring surgical intervention after Gardasil vaccination. Despite many studies demonstrating the excellent results of Gardasil without major side effects, our trial of the Gardasil vaccination (patients 13 and 14) showed no improvement and recurrence was observed shortly thereafter. Similar results were observed in a study done by Hermann et al ${ }^{[15]}$ in 2016, who reported no statistically significant difference in clinical and anatomical scores, surgical intervals and the number of surgeries before and after the Gardasil vacci-nation.

In 2018, Novakovic et al ${ }^{[16]}$ provided the first report of a decline in JORRP incidence in Australia, from 2012 to 2016, which was attributable to the national qHPV vaccination programme. Since 2007, a publicly funded qHPV vaccine has been offered to all schoolgirls aged 12 to 13, with a catch-up programme for older schoolgirls and all women up to age 26. ${ }^{[16]}$ The incidence rate of RRP has declined tremendously from 0.16 per 100,000 in 2012 to 0.02 per 100,000 in 2016. It also confirms, as has been long suspected, that JORRP is a consequence of the transmission of HPV from mother to child around the time of birth. None of the mothers of our JORRP cases received the HPV vaccine before pregnancy, as it is not routinely prac-tised in Malaysia. Fortunately, our centre has not seen any new case of JORRP since 2015, suggesting a falling trend in JORRP cases.

\section{Conclusion}

JORRP remains an upsetting disease to treat, not only due to the significant airway morbidity and risk of recurrence but also due to the emotional and financial strain it brings, and the risk of malignant transformation. Younger onset of JORRP is associated with a worse prognosis. Although there is no definitive cure for RRP, surgery is the mainstay of treatment to provide airway and maintain the voice. Tracheostomy significantly increases the risk of extralaryngeal spread and should be reserved for patients with severe airway compromise after multiple 
surgeries have failed. Adjuvant therapies may be administered for aggressive disease when surgery is insufficient to control the disease, however, there is a lack of a standard protocol.

\section{Acknowledgements: None}

\section{References}

1. Fasunla AJ, Lasisi OA. Diagnostic challenges of laryngeal papillomatosis and its implications among children in developing country. Int $\mathrm{J}$ Pediatr Otorhinolaryngol 2009;73:593-5.

2. Derkay CS, Wiatrak B. Recurrent respiratory papillomatosis: a review. Laryngoscope. 2008;118:1236-47.

3. Gandhi S, Jacob R. Remission in juvenile-onset recurrent respiratory papillomatosis. J Laryngol Voice 2012;2:30-4.

4. Ivancic R, Iqbal H, deSilva B, Pan Q, Matrka L. Current and future management of recurrent respiratory papillomatosis. Laryngoscope Investig Otolaryngol 2018;3:22-34.

5. Lee SM, Park JS, Norwitz ER, et al. Risk of vertical transmission of human papillomavirus throughout pregnancy: a prospective study. PLoS One 2013;8:e66368.

6. Winckworth LC, Nichol R. Question 2: do caesarean sections reduce the maternal-fetal transmission rate of human papillomavirus infection? Arch Dis Child 2010;95:70-3.

7. Omland T, Akre H, Lie KA, Jebsen P, Sandvik L, Brøndbo K. Risk factors for aggressive recurrent respiratory papillomatosis in adults and juveniles. PLoS One 2014;9:e113584.

8. Long YT, Sani A. Recurrent respiratory papillomatosis. Asian J Surg 2003;26:112-6

9. Aaltonen LM, Rihkanen H, Vaheri A. Human papillomavirus in larynx. Laryngoscope 2002;112:700-7.

10. Schraff S, Derkay CS, Burke B, Lawson L. American Society of Pediat-
Ethics Committee Approval: The study was approved by the local ethics committee of the University Kebangsaan Malaysia (UKM PPI/111/8).

Informed Consent: Informed consent was obtained from all individual participants included in the study.

Author Contributions: Designing the study G.B.S.; Collecting the data - G.B.S, S.N.M., L.J.CN; Analysing the data - G.B.S, S.N.M., L.J.CN; Writing the manuscript - G.B.S, S.N.M., L.J.CN; Confirming the accuracy of the data and the analyses - G.B.S.

Conflict of Interest: The authors have no conflicts of interest to declare.

Financial Disclosure: The authors declared that this study has received no financial support.

ric Otolaryngology members' experience with recurrent respiratory papillomatosis and the use of adjuvant therapy. Arch Otolaryngol Head Neck Surg 2004;130:1039-42.

11. Murono S, Nakanishi Y, Tsuji A, et al. Intralesional cidofovir injection for recurrent respiratory papillomatosis in Japan. Auris Nasus Larynx 2016;43:541-5.

12. Pransky SM, Albright JT, Magit AE. Long-term follow-up of pediatric recurrent respiratory papillomatosis managed with intralesional cidofovir. Laryngoscope 2003;113:1583-7.

13. Rogers DJ, Ojha S, Maurer R, Hartnick CJ. Use of adjuvant intralesional bevacizumab for aggressive respiratory papillomatosis in children. JAMA Otolaryngol Head Neck Surg 2013;139:496-501.

14. Kin Cho Goon P, Scholtz LU, Sudhoff H. Recurrent respiratory papillomatosis (RRP)-time for a reckoning? Laryngoscope Investig Otolaryngol 2017;2:184-6.

15. Hermann JS, Weckx LY, Monteiro Nürmberger J, Santos Junior GF, Campos Pignatari AC, Nagata Pignatari SS. Effectiveness of the human papillomavirus (types 6,11,16, and 18) vaccine in the treatment of children with recurrent respiratory papillomatosis. Int J Pediatr Otorhinolaryngol 2016;83:94-8.

16. Novakovic D, Cheng ATL, Zurynski Y, et al. A prospective study of the incidence of juvenile-onset recurrent respiratory papillomatosis after implementation of a national HPV vaccination program. J Infect Dis 2018;217:208-12.

This is an open access article distributed under the terms of the Creative Commons Attribution-NonCommercial-NoDerivs 3.0 Unported (CC BY- NC-ND3.0) Licence (http://creativecommons.org/licenses/by-nc-nd/3.0/) which permits unrestricted noncommercial use, distribution, and reproduction in any medium, provided the original work is properly cited.

Please cite this article as: Goh BS, Shakri NM, Loh JCN. Long Term Outcome of Juvenile Onset Recurrent Respiratory Papillomatosis in University Kebangsaan Medical Centre. ENT Updates 2020;10(1):244-250. 